\title{
Cooling a nanomechanical resonator using feedback: toward quantum behavior
}

\author{
Asa Hopkins ${ }^{1,2}$, Kurt Jacobs ${ }^{2,3}$, Salman Habib² and Keith Schwab4 \\ ${ }^{1}$ Norman Bridge Laboratory of Physics 12-33, California Institute of Technology, Pasadena, \\ CA 91125, USA; \\ ${ }^{2} \mathrm{~T}-8$, Theoretical Division, Los Alamos National Laboratory, Los Alamos, New Mexico 87545, \\ USA; \\ ${ }^{3}$ Centre for Quantum Computer Technology, Centre for Quantum Dynamics, Griffith \\ University, Nathan 4111, Australia; \\ 4Laboratory for Physical Sciences, College Park, Maryland 20740, USA.
}

\begin{abstract}
Nano-electro-mechanical devices are now rapidly approaching the point where it will be possible to observe quantum mechanical behavior. However, for such behavior to be visible it is necessary to reduce the thermal motion of these devices down to temperatures in the millikelvin range. Here we consider the use of feedback control for this purpose. We analyze an experimentally realizable situation in which the position of the resonator is continuously monitored by a Single-Electron Transistor. Because the resonator is harmonic, it is possible to use a classical description of the measurement process, and we discuss both the quantum and classical descriptions. Because of this the optimal feedback algorithm can be calculated using classical control theory. We examine the quantum state of the controlled oscillator, and the achievable effective temperature. Our estimates indicate that with current experimental technology, feedback cooling is likely to bring the required milliKelvin temperatures within reach.
\end{abstract}

Keywords: Nano-electro-mechanics, quantum feedback control, quantum mechanics, continuous measurement, single-electron devices

\section{INTRODUCTION}

In order to observe distinctly quantum dynamical behavior in a macroscopic mechanical resonator, the thermal energy of the oscillator must be sufficiently small with respect to the separation of the quantized energy levels. Up until now it has not been possible to achieve this with real mechanical devices. However, with the development of nano-mechanical resonators which have resonant frequencies in the hundreds of megahertz, and quality factors in the tens of thousands, the observation of quantum behavior is now a real possibility. ${ }^{1,2}$ The temperature which is required to bring the thermal energy of these devices down to a level where quantum effects are observable is on the order of $1 \mathrm{mk}$. Achieving this temperature, however, remains a very difficult task using standard refrigeration technology. It is for this reason that we consider here an alternative method for limiting the thermal fluctuations of a resonator: the use of active feedback control. This involves continuously monitoring the resonator, and using the information to a apply a force in real-time to damp the thermal motion.

A practical method for monitoring the position of the resonator is provided by the use of a Single-ElectronTransitor (SET).$^{3-5}$ A SET consists of two potential barriers which are sufficiently close together that any electrons in the island between the barriers exist only in a set of well-separated discrete energy states. As a result, the precise energy of the highest occupied level has a large effect upon the rate at which electrons can tunnel through the double barrier, and thus affects the current through the SET. An SET can be used to monitor the position of a nanomechanical resonator by placing a charge on the resonator, and locating the resonator close to the island of the SET. Changes in the position of the resonator alter the potential of the island, and thus the current flowing through the SET. The current then provides a continuous read-out of the resonator position.

In order to apply a force to the resonator, we will explicitly consider the use of an "actuating gat", although other methods are just as viable. In this technique a voltage is applied to a gate capacitatively coupled to the

Device and Process Technologies for MEMS, Microelectronics, and Photonics III, J.-C. Chiao,

A. J. Hariz, D. N. Jamieson, G. Parish, V. K. Varadan, Editors, Proceedings of SPIE

Vol. 5276 (2004) @ 2004 SPIE · 0277-786X/04/\$15 · doi: 10.1117/12.522091 
resonator. Changing the gate voltage then changes the applied force. We envisage the situation in which the actuating gate and the SET are situated on opposite sides of the resonator.

In this article we perform a quantum mechanical treatment of the feedback loop using a SET, and evaluate the effectiveness of such a procedure for cooling the resonator. In addition, we explain how the full quantum description of the measurement and feedback process can, for a harmonic resonator, also be modeled exactly using a classical description of measurement and feedback. Thus this article should also be useful for those researchers who wish to know how to treat an observed quantum oscillator without having to use the full quantum formalism. Lastly, we will analyze the quantum state of the resonator which results from the feedback cooling. A natural goal in controlling the motion of a quantum oscillator is to produce "squeezed" states. We describe these states and examine whether the state produced by the feedback algorithm is squeezed.

In Section 2 we describe both the quantum and the equivalent classical description of a simple continuous measurement, and how one can obtain the characteristics of the measurement performed by the SET. In Section 3 we describe the feedback cooling algorithm and analyze its performance, presenting estimates for the steady-state temperatures achievable with current technology. In Section 4 we examine the quantum state of the controlled oscillator in steady-state from the point of view of the production of squeezing. The final section concludes.

\section{MONITORING THE RESONATOR}

\subsection{Classical Continuous Measurement}

To describe a measurement in classical physics, one first determines how the measurement results are related to the measured quantity. This relationship is necessarily probabilistic to take into account the random error in the measurement. An example of such a relationship between a measured quantity $x$ and result $y$ is $y=x+\Delta$ where $\Delta$ is a Gaussian distributed random variable. Here the variance of $\Delta$ determines the measurement accuracy. The purpose of measurement theory is to work out, given the measurement result, what the observer has learned about $x$. This is achieved by using Bayes' theorem, ${ }^{6,7}$ which tells us how the observers initial probability density for $x$ changes as a result of obtaining $y$.

To describe a continuous measurement, one specifies the relationship between the continuous stream of output results, or measurement record, and the observed quantity in each infinitesimal time step $\Delta t$. If we denote the output result in a single time step by $d r$, and the monitored quantity by $y(t)$, then a natural choice is

$$
d r=x(t) d t+\Delta(d t)
$$

where $\Delta(d t)$, being the "noise" on the measurement, is Gaussian distributed. In each interval the measurement result provides some information about the true value of $x$ at that time. In order that the measurement extracts information about $x$ in a continuous fashion, rather than all at once, the information obtained about $y$ must tend to zero as $d t$ tends to zero, and thus the variance of $\Delta(d t)$ must scale in the right way with $d t$. A close examination of the limit reveals that, in fact, this variance must scale as $d t$. The result is that $\Delta(d t)=c d W$, where $c$ is some constant, and $d W$ is an increment of the Gaussian noise process referred to as the Wiener process. (For readers not familiar with the Wiener process, we note that easily accessible treatments may be found in references ${ }^{8}$ and. $^{9}$ ) The equation for $d r$ may therefore be written

$$
d r=x(t) d t+\frac{d W}{\sqrt{8 k}}
$$

where we have defined $k=1 /\left(8 c^{2}\right)$, purely in order to facilitate the discussion in what follows. This is an Ito stochastic equation, so that $d W^{2}=d t .^{8-10}$ Note that $k$ determines, in a loose sense, the rate at which the measurement extracts information about $x$. We will refer to $k$ in what follows as the measurement strength.

Given an initial probability density for $x$, referred to as the observers initial "state of knowledge" regarding the value of $x$, one can derive a (stochastic) equation for the evolution of this state of knowledge as time progresses. This is referred to as the Kushner-Stratonovich equation. ${ }^{11}$ If $x$ is dynamically evolving, and/or driven by noise, then this must be taken into account when deriving the Kushner-Stratonovich equation. It turns out, however, that if the dynamics of $x$ is linear, and the initial state of knowledge for $x$ is given by a Gaussian probability 
density, then the state of knowledge remains Gaussian for all time. Thus, in this case, the Kushner-Stratonovich equation reduces to an equation of motion for the mean and variance of $x$.

The problem we are concerned with here is that in which an observer continuously monitors the position of a damped harmonic oscillator, driven by white noise (this noise will come from various sources, thermal and otherwise). In this case the system has two dynamical variables, the position $x$ and momentum $p$. While it is only the position that is directly observed, the measurement also provides information about the momentum by virtue of the fact that $x$ and $p$ are dynamically coupled. Since the system is linear, the evolution of the observers state of knowledge of the resonator may be written as a set of equations for the evolution of the means and variances of a joint Gaussian distribution for $x$ and $p$.

For reasons which will be explained in what follows, we will need to drive the oscillator with three independent sources of noise. The dynamics of the oscillator will therefore be described by the equations

$$
\begin{aligned}
d x_{\mathrm{c}} & =\frac{1}{m} p_{\mathrm{c}} d t \\
d p_{\mathrm{c}} & =-m \omega_{0}^{2} x_{\mathrm{c}} d t-\Gamma p_{\mathrm{c}} d t+\zeta_{Y} d Y_{\mathrm{c}}+\zeta_{V} d V_{\mathrm{c}}+\zeta_{U} d U_{\mathrm{c}} .
\end{aligned}
$$

Here $m$ is the mass of the oscillator, $\omega_{0}$ is the angular frequency, and $\Gamma$ is the damping rate. The noise sources, $Y$, $V$ and $U$ are all mutually independent Gaussian white noise, so that $\left\langle Y(t) Y\left(t^{\prime}\right)\right\rangle=\left\langle V(t) V\left(t^{\prime}\right)\right\rangle=\left\langle U(t) U\left(t^{\prime}\right)\right\rangle=$ $\delta\left(t-t^{\prime}\right)$. The first noise source $Y$ will be necessary to correctly model noise resulting from the quantum nature of the oscillator, as will be explained in the next section. The second noise source, $V$ is there to allow us to describe the action of the SET, which acts back on the oscillator by driving it with noise which is partially correlated with the the output results. We will denote the correlation coefficient between $V$ and the measurement noise $W$ as $\kappa$, so that $\left\langle V(t) W\left(t^{\prime}\right)\right\rangle=\kappa \delta\left(t-t^{\prime}\right)$. The final noise source, $U$, represents the noise from the thermal bath, and the expression for $\zeta_{U}$ will be given in the next section.

If the position of the oscillator is continually observed using the measurement given by Eq.(2), then the observers state of knowledge is characterized by the means and covariances of $x$ and $p$. The equations for the means, which are the observers best estimates of $x$ and $p$, are

$$
\begin{aligned}
& d\langle x\rangle=\frac{\langle p\rangle}{m} d t+2 \sqrt{2 k} \sigma_{x}^{2} d W \\
& d\langle p\rangle=-m \omega^{2}\langle x\rangle d t-\Gamma\langle p\rangle d t-\gamma(m \omega\langle x\rangle+\langle p\rangle) d t+\sqrt{\kappa} \zeta_{V} d W+2 \sqrt{2 k} \sigma_{x p}^{2} d W
\end{aligned}
$$

The equations for the covariances are

$$
\begin{aligned}
\dot{\sigma}_{x}^{2} & =\frac{2}{m} \sigma_{x p}^{2}-8 k\left(\sigma_{x}^{2}\right)^{2} \\
\dot{\sigma_{p}^{2}} & =-2 m \omega^{2} \sigma_{x p}^{2}-8 k\left(\sigma_{x p}^{2}\right)^{2}-2 \Gamma \sigma_{p}^{2}+\zeta_{Y}^{2}+(1-\kappa) \zeta_{V}^{2}+\zeta_{U}^{2} \\
\dot{\sigma_{x p}^{2}} & =\frac{\sigma_{p}^{2}}{m}-m \omega^{2} \sigma_{x}^{2}-\frac{\Gamma}{2} \sigma_{x p}^{2}-8 k \sigma_{x}^{2} \sigma_{x p}^{2}-\sqrt{8 \kappa k} \zeta_{V} \sigma_{x}^{2} .
\end{aligned}
$$

In these equations, $\sigma_{x}^{2}$ and $\sigma_{p}^{2}$ are the variances of the position and momentum, respectively, and $\sigma_{x p}^{2} \equiv\langle x p\rangle-$ $\langle x\rangle\langle p\rangle$ is the covariance between $x$ and $p$.

Given the measurement record (Eq.(2)), the observer can obtain $d W$ at each time-step, and integrate the above equations, which provide her best estimates of $x$ and $p$ as time progresses. The observer can then implement feedback by applying a force to the oscillator as a function of the estimates $\langle x\rangle$ and $\langle p\rangle$. In fact, modern classical control theory tells us that for linear systems with linear feedback, the optimal control algorithm can always be achieved using feedback which is a function of the best estimates.

The performance of a feedback algorithm can then be evaluated by including the feedback dynamics in Eq(6) (since the evolution of the variances is not affected by the application of a linear force), and solving. While the analysis we have performed in the section is purely classical, it is useful because, as we will show in the next section, it is sufficient to describe the full quantum dynamics, so long as we choose the noise sources appropriately. 


\subsection{Quantum Continuous Measurement}

Orthodox quantum measurement theory may be viewed as the quantum equivalent of Bayesian statistical inference. To describe a continuously observed quantum system, various approaches are possible, but a simple method is to apply quantum measurement theory in a similar way to that described above for the derivation of a classical continuous measurement. (While there do not appear to be any full examples of this in the literature to date, treatments along these lines are given in Refs. $\left.{ }^{13,14}\right)$. In the quantum case, the observers state-of-knowledge of the quantum resonator is now given by the quantum density matrix, $\rho$, and the equation which describes the evolution of the density matrix (being the quantum equivalent of the Kushner-Stratonovich equation), is referred to as a Stochastic Master Equation (SME). ${ }^{16,17}$

In the classical case, the measurement record was the actual value of the observed quantity, with the addition of Gaussian noise. In the quantum case, there is no "rea" value of the observable. However, one can rewrite the classical measurement record in way which has a direct quantum analogue. This is

$$
d r=\langle x(t)\rangle d t+\frac{d \xi}{\sqrt{8 k}},
$$

where $\langle x(t)\rangle$ is the expectation value of the observable given by the observers current state-of-knowledge, and $d \xi$ is Gaussian white noise, uncorrelated with $\langle x(t)\rangle$. The measurement record in the quantum case is obtained simply by replacing the classical average $\langle x(t)\rangle$ with the quantum expectation value of the observable, $\langle x(t)\rangle=\operatorname{Tr}[\rho x]$, where $\rho$ is the quantum density matrix.

The SME must include the dynamics of the damped resonator. The damping of the resonator is a result of its interaction with its environment, which we will model as a bath of harmonic oscillators, in the usual manner of Caldiera and Leggett. ${ }^{18}$ The result of this is the Standard Brownian Motion Master Equation (SBMME). Since the resonators with which we are concerned have high quality factors, we will use an approximate form of the SBMME which is valid for the weak coupling regime (weak damping), but covering all ranges of temperatures. ${ }^{19}$

The SME which describes the evolution of the quantum density matrix for the resonator under continuous measurement of the position is ${ }^{20}$

$$
\begin{aligned}
d \rho= & -\frac{i}{\hbar}[H, \rho] d t-\frac{i \Gamma}{2 \hbar}[x, p \rho+\rho p] d t-\left(k+\beta+\frac{m \omega_{0} \Gamma}{2 \hbar} \operatorname{coth} \frac{\hbar \omega_{0}}{2 k_{B} T}\right)[x,[x, \rho]] d t \\
& -i \sqrt{2 \kappa \beta}[x, \rho] d W+\sqrt{2 k}(x \rho+\rho x-2\langle x\rangle \rho) d W .
\end{aligned}
$$

The first term gives the deterministic motion due to the harmonic Hamiltonian

$$
H=\frac{p^{2}}{2 m}+\frac{1}{2} m \omega_{0}^{2} x^{2}
$$

The second term gives the damping at rate $\Gamma$ due to the interaction with the thermal bath. The third term describes the momentum diffusion from three sources of white noise. The first is the necessary quantum backaction from the continuous measurement of position with measurement strength $k$. The second, with strength given by $\beta$, is noise over and above the necessary back-action (this noise will be due to the action of the SET, as will be explained later). The third source of white noise is that due to the thermal bath. The cotangent function which appears in the expression for the strength of this noise is there to describe correctly the fact that even at zero temperature there is still noise from the quantum bath.

The forth and fifth terms in Eq.(10) give the update in the observers state-of-knowledge as a result of the continuous measurement. The forth term is the effect of allowing the noise source $\beta$ to be correlated with the noise on the measurement record, with correlation co-efficient $\kappa$. The final term is thus the update in the absence of any correlation.

If the initial density matrix for the system is Gaussian in position, then the SME preserves this Gaussianity as time progresses. Consequently, just like the classical Kushner-Stratonovich equation, it may be replaced by a set of equations for the means and covariances of $x$ and $p$. What is more, the equation for the means and 
covariances are precisely the same as the classical equations introduced in the previous section, so long as we make the following identifications:

$$
\begin{aligned}
\zeta_{Y} & =\sqrt{2 \beta} \hbar, \\
\zeta_{V} & =\sqrt{2 k} \hbar, \\
\zeta_{U} & =\sqrt{\hbar m \omega_{0} \Gamma \operatorname{coth} \frac{\hbar \omega_{0}}{2 k_{B} T}} .
\end{aligned}
$$

As a result, one can correctly describe the dynamics of a continuously monitored, quantum resonator, subject to noise from a quantum heat bath, by using a classical model, so long as one chooses the noise terms in the model correctly. In particular, one must include the essential quantum back-action from the continuous measurement by adding a white noise force proportional to $\hbar$ and $\sqrt{k}$.

\subsection{Modeling the SET}

Treatments of the continuous measurement of a charge qubit using a $\mathrm{SET}^{14,15}$ make it clear that, at least to a good approximation, the SET provides a continuous measurement of the type discussed above. This is to be expected, since this form is rather canonical - it does not depend on the precise physics of the measuring apparatus, but on the Gaussian relationship between the observed quantity and the measurement results. Due to the central limit theorem, most continuous measurements which provide a continuous (as opposed to a point process) output record are Gaussian.

In order to treat the SET measurement, we thus need to calculate $k$, and if there is any excess noise, $\beta$ and $\kappa$ for the SET. The simplest way to do this is to note that the spectra of the current through an SET coupled to a resonator has already been calculated by Zhang and Blencowe, ${ }^{21}$ based on a previous analysis by Korotkov. ${ }^{14}$ One merely needs to calculate the spectrum of the measurement results determined by the stochastic master equation, and equate this with the spectra obtained by Zhang and Blencowe. This then provides us with expressions for $k$ and $\beta$ in terms of this spectra. In particular one has

$$
k=\frac{(d I / d x)^{2}}{\left.8 S_{I}(\omega)\right|_{\omega=0}},
$$

where $I$ is the current through the SET, $d I / d x$ is the constant of proportionality between fluctuations in $I$ and those in the oscillator position, and $S_{I}(\omega)$ is the (shot noise) spectrum of the current fluctuations. For $\beta$ the expression is

$$
\beta=\left.\frac{S_{\mathrm{F}}(\omega)}{2 \hbar^{2}}\right|_{\omega=0}
$$

where $S_{\mathrm{F}}$ is the spectrum of fluctuations of the force on the resonator due to the fluctuations of the charge on the SET island, calculated using the semi-classical treatment. (The semi-classical treatment does not include the quantum back-action, hence $S_{\mathrm{F}}$ contributes to $\beta$ only, not to $k$ ). The correlation coefficient, $\kappa$, between the shot noise and the excess back action is simply the correlation coefficient between $S_{I}$ and $S_{\mathrm{F}}$. The expressions for the spectra are fairly complicated, but may be found in Refs. ${ }^{21}$ and,${ }^{20}$ the second of which also gives the correlation spectrum.

Finally, it is important to note that the semi-classical analysis which we use to obtain the spectra is only valid in certain parameter regimes. The first two requirements, that of low temperature and low drain-source voltage $^{3,20,21}$ are not of undue concern. However, the semi-classical analysis, since it only gives the excess noise on the resonator, rather than the full noise, cannot necessarily be trusted when $k$ is not much smaller than $\beta$. Further, the ratio $k / \beta$ is not guaranteed to be bounded by any given value in the regime of best cooling. Thus, when we present results in what follows, we will give both the best predicted cooling, and that under the restriction that $k \leq \beta / 10$. 


\section{FEEDBACK COOLING}

We wish to cool the resonator by applying a linear force, which is continuously altered in response to the information provided by the continuous measurement - that it, by applying a feedback force. Since the oscillator can be described by a linear classical model, modern optimal control theory can be used to obtain an optimal feedback algorithm for cooling the resonator. In the limit in which the feedback force is sufficiently strong, the optimal algorithm involves choosing the force to be $\mathrm{b}^{12}$

$$
F=-\gamma\left(m \omega_{0}\langle x\rangle+\langle p\rangle\right)
$$

where $\gamma$ is a rate constant which determines the overall strength of the force. This equation gives optimal performance so long as $\gamma \gg \omega_{0}$, which is within reach of current experiments, as detailed below. To implement the algorithm, the measurement record is used to integrate the equations of motion for the observers state-ofknowledge in real time, which provides the best estimates of the position and momentum, being $\langle x\rangle$ and $\langle p\rangle$, and then the force is determined by Eq.(17).

To evaluate the performance of the feedback loop, we solve for the steady-state of the oscillator under feedback. The addition of the feedback force does not affect the equations of motion for the covariances, so first we solve these equations for the steady-state. Since the quality factor of the the resonator is high, it is reasonable to assume that $\Gamma$ is much smaller than $\gamma$, being the damping rate associated with the feedback force. We thus simplify the equations for the covariances by dropping the damping terms proportional to $\Gamma$, making them much simpler to solve. The solution is

$$
\begin{aligned}
\sigma_{x}^{2} & =\frac{\sqrt{2} \omega}{8 k} \sqrt{\Lambda} \\
\sigma_{p}^{2} & =\frac{\sqrt{2} m^{2} \omega^{3}}{8 k}\left[\sqrt{\Lambda}+\Lambda^{3 / 2}\right]+\frac{\hbar m \omega}{\sqrt{2 k}} \sqrt{\kappa \beta} \sqrt{\Lambda}, \\
\sigma_{x p}^{2} & =\frac{m \omega^{2}}{8 k} \Lambda,
\end{aligned}
$$

where

$$
\Lambda+1=\left[1+\frac{16 k \hbar^{2}}{m^{2} \omega^{4}}\left\{k+(1-\kappa) \beta+\frac{m \omega_{0} \Gamma}{2 \hbar} \operatorname{coth} \frac{\hbar \omega_{0}}{2 k_{B} T}\right\}\right]^{1 / 2} .
$$

In the limit of both large and small values of $k, \Lambda \sim k$.

However, the steady-state fluctuation in the means also contributes to the average value of the energy of the oscillator. To calculate the steady-state variances of the means, we substitute the steady-state values for the variances into $\operatorname{Eqs}(5)$ and (6), include the effect of the force in Eq.(6), and solve. The resulting variances for the means (in steady-state) are

$$
\begin{aligned}
\sigma_{\langle x\rangle}^{2}= & \frac{\omega\left(\gamma^{2}+\gamma \omega+\omega^{2}\right)}{8 k \gamma(\omega+\gamma)} \Lambda+\frac{\sqrt{2} \omega^{2}}{8 k(\omega+\gamma)} \Lambda^{3 / 2}+\frac{\omega^{3}}{16 k \gamma(\omega+\gamma)} \Lambda^{2} \\
& +\frac{\kappa \beta \hbar^{2}}{m^{2} \omega \gamma(\omega+\gamma)}+\frac{\hbar \sqrt{\kappa k \beta}}{2 m k(\omega+\gamma)}\left[\sqrt{2 \Lambda}+\frac{\omega \Lambda}{2 \gamma}\right] \\
\sigma_{\langle p\rangle}^{2}= & \frac{m^{2} \omega^{3}(\omega+\gamma)}{8 k \gamma} \Lambda+\frac{m^{2} \omega^{4}}{16 k \gamma} \Lambda^{2}+\frac{\kappa \beta \hbar^{2}}{\gamma}+\frac{m \omega^{2} \hbar \sqrt{\kappa \beta k}}{4 k \gamma} \Lambda .
\end{aligned}
$$

The average energy of the oscillator in the steady-state is then

$$
\begin{aligned}
E & =\langle H\rangle=\frac{1}{2} m \omega^{2}\left(\sigma_{x}^{2}+\sigma_{\langle x\rangle}^{2}\right)+\frac{\sigma_{p}^{2}+\sigma_{\langle p\rangle}^{2}}{2 m} \\
& =\frac{m \omega^{3}}{8 k}\left[\sqrt{2 \Lambda}+\Lambda+\frac{\sqrt{2}}{2} \Lambda^{3 / 2}+\frac{\omega}{4 \gamma} \Lambda^{2}\right]+\frac{\kappa \beta \hbar^{2}}{2 m \gamma}+\frac{\hbar \omega \sqrt{\kappa \beta k}}{4 k}\left[\sqrt{2 \Lambda}+\frac{\omega}{2 \gamma} \Lambda\right] .
\end{aligned}
$$




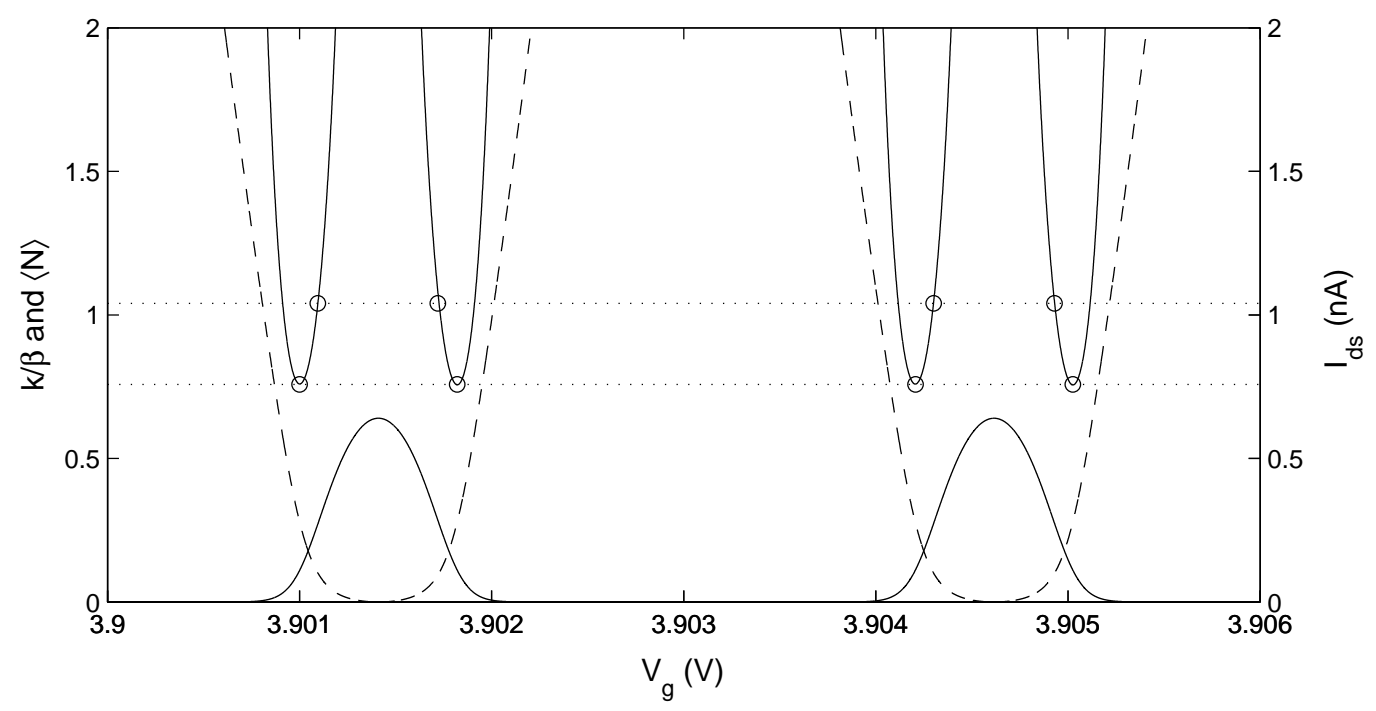

Figure 1. The steady-state average occupation number, $\langle N\rangle$, as a function of the gate voltage (upper solid line), plotted along with the ratio $k / \beta$ (dashed line), and the drain-source current, $I_{\mathrm{d} s}$ (lower solid line). The lower dotted line gives the minima of $\langle N\rangle$, and the upper dotted line gives the values of $\langle N\rangle$ when $k / \beta=0.1$.

In the final expression above we have made the simplifying assumption that $\gamma \gg \omega$, since this is inherent in the optimal control condition. We now evaluate the achievable energy for realistic experimental parameters. In doing so we will quote the results mainly in terms of the final temperature. Of course, in the steady-state, the resonator is actually far from equilibrium. Thus, the temperature which we will quote is actually an effective temperature, being the temperature which an oscillator in thermal equilibrium would have if it had the same average energy.

Current refrigeration technology allows experiments on nanomechanical resonators to be performed at temperatures of about $100 \mathrm{mK}$. We will thus assume that the feedback algorithm will be applied to a device initially at this temperature. Typical fundamental frequencies for resonators in these experiments fall in the range $f_{0}=1-100 \mathrm{MHz}$. As our example system we take a realistic resonator with $f_{0}=12 \mathrm{MHz}$, which is $6 \mu \mathrm{m}$ in length, $50 \mathrm{~nm}$ wide, and $150 \mathrm{~nm}$ thick. We restrict ourselves to relatively low frequencies because of the limits of feedback circuitry, which we estimate can easily operate at $50 \mathrm{MHz}$. The effective mass of such a resonator is roughly $10^{-16} \mathrm{~kg}$, and an achievable quality factor, $Q$, is on the order of $10^{4}$.

Realistic values for the resistances and capacitances of the junctions of a SET which would be used to monitor the resonator are $R_{1}=R_{2}=50 \mathrm{k} \Omega$, and $C_{1}=C_{2}=100 \mathrm{aF}$, and we place it $d \sim 100 \mathrm{~nm}$ from the resonator. We estimate that the capacitance between the gate of the SET and the resonator will be roughly $C_{\mathrm{g}}=50 \mathrm{aF}$, so that $C_{\Sigma}=250 \mathrm{aF}\left(C_{\Sigma}=2 C_{j}+C_{\mathrm{g}}\right)$. It is important to note that our treatment of the SET is only a good approximation so long as the drain-source voltage across the SET, $V_{\mathrm{ds}}$, satisfies $V_{\mathrm{ds}} \ll e / C_{\Sigma}$, and $k / \beta \ll 1$, as was discussed in Section 2.3.

To apply the feedback force, we place the resonator $100 \mathrm{~nm}$ from the actuating gate, and allow the controller to vary the voltage difference between the gate and the resonator between $-4 \mathrm{~V}$ and $4 \mathrm{~V}$. The capacitance of this arrangement is about $50 \mathrm{aF}$, so the maximum force that can be applied to the resonator is around $10^{-8} N$. This corresponds to $\gamma \approx 1.08 \times 10^{13} \mathrm{~s}^{-1}$, which is much larger than $\omega$ and $\Gamma$, as required by the optimal control condition and the small $\Gamma$ approximation used above.

Before giving theoretical estimates for the achievable steady-state effective temperature (or equivalently, the steady-state average occupation number of the oscillator, $\langle N\rangle=\left\langle a^{\dagger} a\right\rangle$ ), we need to explain two subtleties which affect the presentation of our results. When one examines the dependence of the steady-state $\langle N\rangle$ on the gate voltage, one finds that it oscillates very rapidly, with minima occurring in closely spaced pairs. Since $V_{\mathrm{g}}$ is 

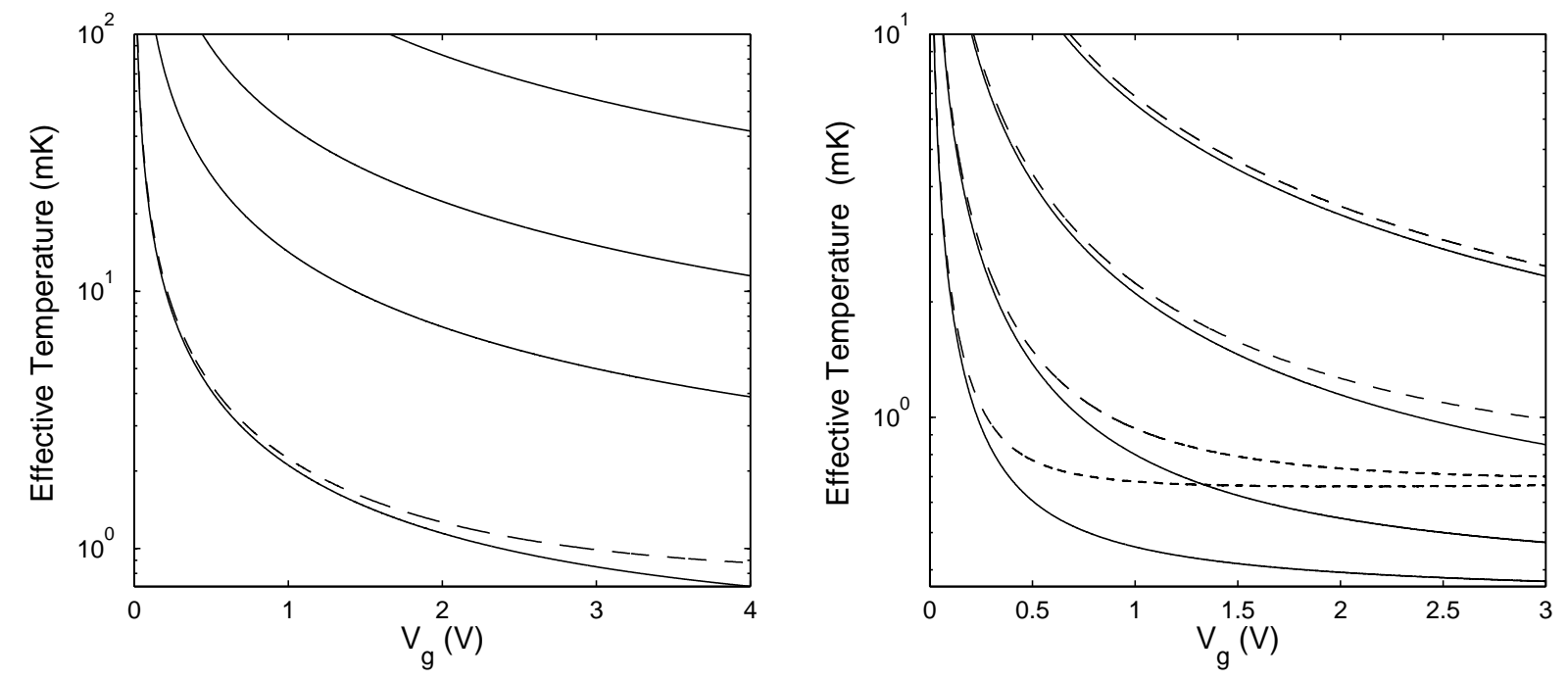

Figure 2. (a) Estimates for the minimum achievable effective temperatures as a function of gate voltage for a range of initial temperatures, $T$. For $T=100 \mathrm{mK}$ the dashed line shows the result under the restriction that $k / \beta \leq 0.1$. For larger values of $T$ the effect of this restriction is negligible on this plot. From top to bottom, the initial temperatures are $2 \mathrm{~K}, 1 \mathrm{~K}, 500 \mathrm{mK}$, and $100 \mathrm{mK}$. (b) Minimum temperatures as in (a), but this time for a range of $Q$ factors, all at an initial temperature $T=100 \mathrm{mK}$. The dashed lines give the minimum temperature under the additional restriction that $k / \beta \leq 0.1$. From top to bottom, the quality factors are $10^{3}, 10^{4}, 10^{5}$, and $10^{6}$. A quality factor of at least $10^{4}$ is achievable with current technology.

experimentally easy to tune, all else being equal it would make sense simply to plot these minima and ignore the complex structure. However, as discussed in section 2.3, our results are more trustworthy the smaller $k / \beta$, and this quantity is not necessarily small at the minima. The situation is shown in detail in Figure 1, in which we display, as a function of $V_{\mathrm{g}}$, two pairs of the $\langle N\rangle$ minima, as well as $k / \beta$ and the current $I_{\mathrm{ds}}$. In view of this, when plotting results in what follows, we will show both the minima of the effective temperature with respect to $V_{\mathrm{g}}$, and the (somewhat higher) effective temperature which results if we demand that $k / \beta \leq 0.1$. For clarity the points at which $k / \beta=0.1$ are also displayed in Figure 1. As will be clear from figure 2 , for $T=100 \mathrm{mK}$ and $Q=10^{4}$, the effect of the restriction $k / \beta \leq 0.1$ on the achievable temperature is small. In addition, $k / \beta$ remains fairly small at the minima. Since this is the case, when we quote values in the following, we will give the values obtained at the minima, along with the corresponding values for $k / \beta$.

As an example of the relative magnitudes of the various noise sources at the energy minima, if we set the drain-source voltage at $V_{\mathrm{ds}}=e /\left(4 C_{\Sigma}\right)=0.16 \mathrm{mV}$ and the gate voltage at $V_{\mathrm{g}} \sim 1 \mathrm{~V}$, then the noise sources are: $\beta=1.01 \times 10^{31} \mathrm{~m}^{-2} \mathrm{~s}^{-1}, k=0.184 \beta, \frac{m \omega_{0} \Gamma}{2 \hbar} \operatorname{coth} \frac{\hbar \omega_{0}}{2 k_{B} T}=9.25 \beta$ and the correlation coefficient is $\kappa=0.638$.

Using the above parameter values to calculate the effective temperature, $T_{\text {eff, }}$, at the minima, we find that $\Lambda=5.1 \times 10^{-5}$, and $T_{\text {eff }}=2.11 \mathrm{mK}$. This corresponds to an energy of about $E_{\mathrm{ss}}=2.91 \times 10^{-26} \mathrm{~J}$, and an average occupation number $\langle N\rangle=3.17$. While this is very encouraging, ideally one wants to cool below the energy of the first excited state. If we increase the strength of the measurement (i.e. the gate voltage $V_{\mathrm{g}}$ ), then we find that this does lead to better cooling. However, the returns eventually diminish with increasing $V_{g}$, as a result of the increased in the quantum back-action. In addition, at some value of $V_{\mathrm{g}}$ snap-in is likely to occur as the force between the SET gate and the resonator becomes too strong. This voltage, in our example system, is estimated to be roughly $4 \mathrm{~V}$. At $V_{\mathrm{g}}=4 \mathrm{~V}$ the steady state minimum energy $E=9.83 \times 10^{-27} \mathrm{~J}$, which is below the energy of the first excited state. This corresponds to $T_{\text {eff }}=0.71 \mathrm{mK}$ and $\langle N\rangle=0.74$, with $k / \beta=0.28$. Thus, if the energy were to be measured directly, immediately after turning off the feedback, energy jumps as a signature of quantum behavior may well be observable. As an indication of the return from increasing the gate 
voltage, the minimum steady state energy is $E=1.58 \times 10^{-26} \mathrm{~J}$ for $V_{\mathrm{g}} \approx 2 \mathrm{~V}$, which corresponds to $\langle N\rangle \approx 1.5$, with $k / \beta=0.21$.

In Figure 2 (a) we plot the theoretical estimates for the achievable steady-state effective temperature as a function of $V_{\mathrm{g}}$ for a range of starting temperatures. The solid lines correspond to the absolute minima, and the dotted lines to the minimum values under the restriction that $k / \beta \leq 0.1$. Of particular interest is the fact that for a starting temperature of $2 \mathrm{~K}$ (ie. with pumped liquid $\mathrm{He}$ ), we obtain minimum temperatures in the range of $50 \mathrm{mK}$. Thus, even for an initial temperature of $2 \mathrm{~K}$, feedback cooling might well be able to compete with dilution refrigerators. If the resonator is first cooled in a dilution refrigerator, and then feedback cooled, the semi-classical theory predicts achievable temperatures below $1 \mathrm{mK}$, as discussed above. In Figure 2 (b) we plot the dependence of the minimum temperature on $V_{\mathrm{g}}$ for a range of quality factors, which shows that somewhat lower final temperatures could be achieved by increasing $Q$.

\section{THE QUANTUM STEADY-STATE}

It is also of interest to examine the full quantum state of the resonator which is produced by the feedback algorithm. This is because one of the first challenges in controlling a quantum resonator will be to produce squeezed states (to be discussed below). In addition, once the resonator is cooled, the observation of quantum behavior will ultimately involve preparing the resonator in non-classical states, either by applying a non-linear Hamiltonian, or the measurement of an operator which is not linear in $x$ and $p$.

As discussed in Section 2.2, this is a state with a Gaussian Wigner function, and thus characterized by the means and covariances of position and momentum. The famous Heisenberg uncertainty relation states that $\sigma_{x} \sigma_{p} \geq \hbar / 2$. In considering the variances of $x$ and $p$ it is useful for us to work with scaled units, in which the position and momentum are scaled by their respective standard deviations in the ground state of the resonator. Thus we define scaled position and momentum operators $X$ and $P$ by

$$
\begin{aligned}
& X=x \sqrt{\frac{2 m \omega}{\hbar}}, \\
& P=p \sqrt{\frac{2}{m \omega \hbar}} .
\end{aligned}
$$

With this definition, Heisenberg's uncertainty relation becomes $\sigma_{X} \sigma_{P} \geq 1$.

If the state is such that either of the standard deviations of $X$ or $P$ are less than unity (or more generally, if there exists a linear combination of $X$ and $P$ which has a variance less than unity), then the state is said to be squeezed. ${ }^{16}$ One of the first challenges in controlling the state of a nano-mechanical resonator will be to produce squeezed states. Since the linear feedback of our cooling algorithm does not effect the variances (just the mean values), the variances are determined purely by the interplay between the measurement, the harmonic oscillation, and the environmental noise.

A continuous measurement of position can, in fact, produce a squeezed state: if one considers a very strong measurement performed over a short time, then the position variance is reduced at the expense of the momentum variance, and the state is squeezed. However, the resonator is a harmonic oscillator, and harmonic motion is simply a rotation of the system in phase space at angular frequency $\omega$. Thus, a state which is squeezed in position will be rotated by the evolution so that it is periodically, and alternately, squeezed in position and momentum. As a result, a continuous measurement of position will be periodically working to squeeze, and then unsqueeze, the state. If the rotation is fast compared to the rate at which position information is extracted, then the steady-state has very little squeezing. In the case where there is no environmental noise, so that the state is pure, the parameter which determines the amount of squeezing in the steady state is $r=4 k \hbar /\left(m \omega^{2}\right) .{ }^{22,23}$ If $r$ is large there is significant squeezing.

In our case there is also environmental noise, which increases the variance of both $X$ and $P$. Specifically, from $\operatorname{Eqs}(18)-(20)$ we have

$$
\delta X^{2}=\sqrt{2}\left(\frac{1}{r}\right) \sqrt{\sqrt{1+(1+c) r^{2}}-1}
$$




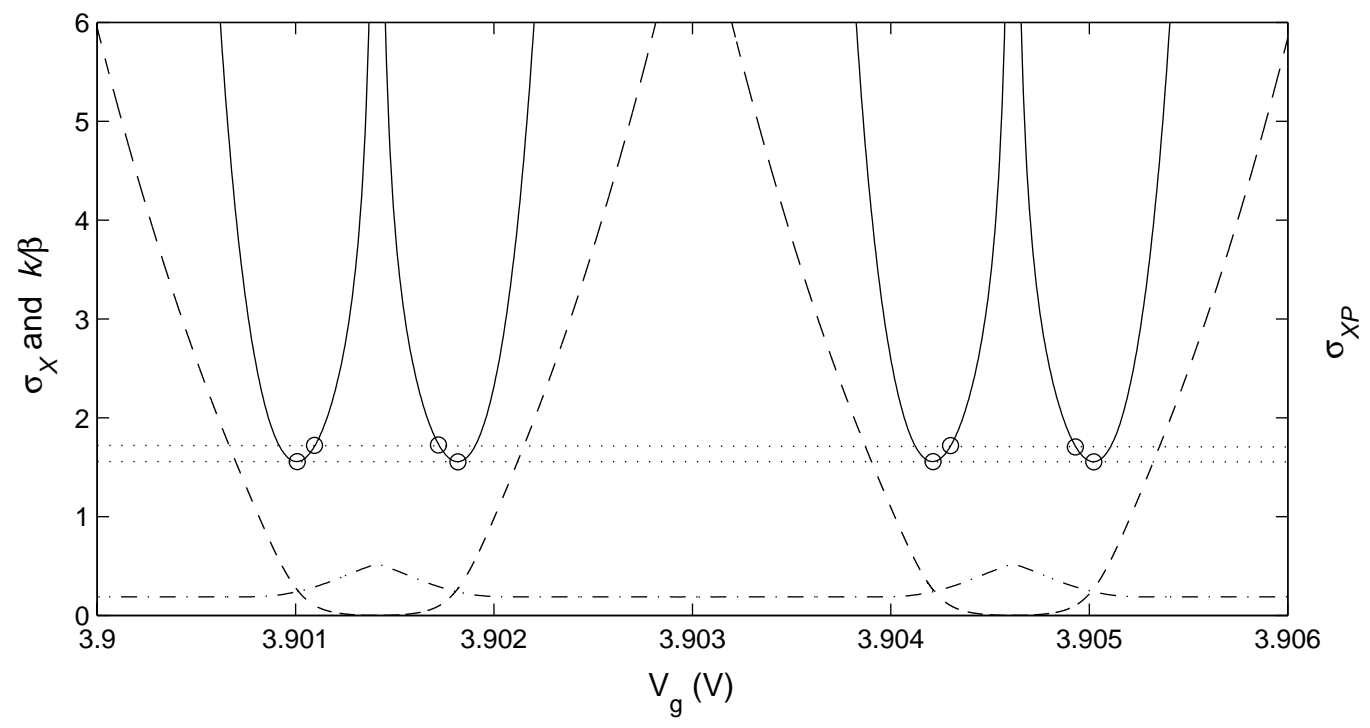

Figure 3. The standard deviation of the scaled position, $\sigma_{X}$, as a function of the gate voltage (solid line). The standard deviation of the scaled momentum is almost identical. We also plot the ratio $k / \beta$ (dashed line), and the scaled positionmomentum covariance, $\sigma_{X P}$ (dot-dash line). The lower dotted line gives the minima of $\sigma_{X}$, and the upper dotted line gives the values of $\sigma_{X}$ when $k / \beta=0.1$.

$$
\delta P^{2}=\delta X^{2} \sqrt{1+(1+c) r^{2}}+\sqrt{\frac{2 \kappa \beta}{k}} \sqrt{\sqrt{1+(1+c) r^{2}}-1}
$$

where $c$ is a dimensionless number giving the ratio of the excess environmental noise to the measurement noise:

$$
c=\frac{(1-\kappa) \beta+\frac{m \omega_{0} \Gamma}{2 \hbar} \operatorname{coth} \frac{\hbar \omega_{0}}{2 k_{B} T}}{k} .
$$

If $r$ is not sufficiently large (that is, if the measurement is not sufficiently strong), and the environmental noise is sufficiently strong, then the oscillator will not be squeezed. In addition, it should be noted that the means of $X$ and $P$ are also fluctuating in the steady-state. It is therefore sensible, in defining the notion of squeezing, to include these fluctuations in the overall variances of $X$ and $P$. However, with the parameters considered here, the effect of adding the variances of $\langle X\rangle$ and $\langle P\rangle$ to the quantum variances has only a very minor effect.

In figure 3 we plot the standard deviations of $X$ and $P$, along with the square root of the covariance, for the largest reasonable values of $V_{\mathrm{g}}$, and hence the strongest available measurement. From this we see that the measurement is not sufficient to squeeze the oscillator in the presences of the excess noise. Of course, to look for squeezing one really needs to take the linear combination of $X$ and $P$ which has the minimum variance however, in our case this minimum variance is only marginally smaller that the position variance, because the covariance is small.

However, it is possible to use a more sophisticated approach if one wishes to produce squeezing. If one measures a linear combination of $x$ and $p$ which rotates in phase space at the frequency of the oscillator, then such a measurement never works against itself from the point of view of producing squeezing. If one works in radial coordinates $(A, \theta)$ in phase space, so that $X=A \cos \theta$ and $P=A \sin \theta$, then such a measurement will produce squeezing at some constant direction with respect to these coordinates. For an ideal resonator, this will produce amplitude or phase squeezing, for example. In the present situaton although one cannot measure $p$, one could consider turning off the position measurement over those times in the cycle when it will be working against the squeezing. ${ }^{24}$ Ruskov, Schwab and Korotkov have recently analyzed such a scheme for a realistic SET measurement. ${ }^{25}$ 


\section{CONCLUSION}

We have shown here that quantum feedback control could be an effective means of cooling nano-mechanical devices below that achievable with other technology, such as dilution refrigerators, and in particular for achieving the level of control required for the observation of quantum behavior. More generally, feedback control is likely to be an important technique for cooling and controlling quantum nano-mechanical systems.

In performing our analysis we have, of course, made certain simplifying assumptions. In a real experimental implementation one would have a finite bandwidth detector, with some level of inefficiency, and a certain amount of noise and delay time in the classical feedback circuit. Nevertheless the implementation of feedback using SET's certainly appears to be within the ability of current technology, and the present work indicates that this is a goal worth pursuing.

\section{REFERENCES}

1. A.N. Cleland and M.L. Roukes, Appl. Phys. Lett. 69, 2653 (1996).

2. See, e.g., A.D. Armour, M.P. Blencowe, and K.C. Schwab, Phys. Rev. Lett. 88, 148301 (2002).

3. A.N. Korotkov, Phys. Rev. B 49, 10381 (1994).

4. U. Hanke et al., Appl. Phys. Lett. 65, 1847 (1994).

5. A. Shnirman and G. Schön, Phys. Rev. B 57, 15400 (1998).

6. E.T. Jaynes, in E.T. Jaynes : Papers on Probability, Statistics, and Statistical Physics, edited by R.D. Rosenkrantz, (Dordrecht, Holland, 1983).

7. S.J. Press, Bayesian Statistics : Principles, Models, and Applications, (Wiley, New York, 1989).

8. D.T. Gilespie, Am. J. Phys. 64, 225 (1996).

9. K. Jacobs, PhD thesis, Imperial College, London (unpublished), Eprint: quant-ph/.

10. C. W. Gardiner, 'Handbook of Stochastic Methods' (Springer-Verlag, Berlin, 1985).

11. P.S. Maybeck, Stochastic Models, Estimation and Control, volumes II and III, (Academic Press, New York, 1982).

12. A.C. Doherty and K. Jacobs, Phys. Rev. A 60, 2700 (1999).

13. C.M. Caves and G.J. Milburn, Phys. Rev. A 36, 5543 (1987).

14. A.N. Korotkov, Phys. Rev. B 60, 5737 (1999); A.N. Korotkov, Phys.Rev.B 63, 115403 (2001).

15. N. Oxtoby, H.M. Wiseman, P. Warszawski and R. Polkinghorne, (in preparation).

16. H.M. Wiseman and G.J. Milburn, Phys. Rev. A 47, 642 (1993).

17. A.C. Doherty, S. Habib, K. Jacobs, H. Mabuchi and S.M. Tan, Phys. Rev. A 62012105 (2000).

18. A.O. Caldeira and A.J. Leggett, Physica A 121587 (1983).

19. A.O. Caldeira, H.A. Cerdeira, and R. Ramaswamy, Phys. Rev. A 40, 3438 (1989).

20. A. Hopkins, K. Jacobs, S. Habib and K. Schwab, PRB (in press).

21. Y. Zhang and M.P. Blencowe, J. App. Phys. 91, 4249 (2002), Eprint: cond-mat/0109412.

22. V. P. Belavkin and P. Staszewski, Phys. Rev. A 45 (1992).

23. K. Jacobs and P.L. Knight, Phys. Rev. A. 57, 2301 (1998).

24. V.B. Braginsky and F.Ya. Khalili, Phys. Lett. A 175, 85 (1993); Rev. Mod. Phys. 681 (1996).

25. R. Ruskov, K.C. Schwab and A.N. Korotkov, (in preparation). 